\title{
Ultrasound-guided bilateral erector spinae plane block versus intrathecal morphine for analgesia in living donors for liver transplantation: A randomized noninferiority trial
}

\section{RyungA Kang,1 Duck Hwan Choi,1 Justin Sangwook Ko,1 Han Bum Jo,2 Jin Soo Kim2}

From the department of Anesthesiology and Pain Medicine, Samsung Medical Center, Sungkyunkwan University School of

Medicine, Seoul, Korea

Department of Anesthesiology and Pain Medicine, Ajou

\section{Background}

- Preoperative ITM administration is an effective analgesic method in living donor hepatectomy.

- But, is associated with adverse effects, including postoperative nausea, vomiting, and pruritus.

- The ESP block has been shown to provide effective postoperative analgesia after various laparoscopic abdominal surgeries.

- We hypothesized that it would provide noninferior postoperative analgesia compared to ITM and reduce postoperative nausea/vomiting and pruritus.

\section{Materials \& Methods}

- A non-inferiority trial

- Fifty-four patients scheduled for pure laparoscopic right donor hepatectomy were randomly assigned to one of two groups: an ESP group $(n=27)$ and an ITM group ( $n=27)$.

- ESP group received preoperative bilateral single-injection ESP blocks at the level of the T8 transverse process with an injection of 20 $\mathrm{mL} 0.5 \%$ ropivacaine with $5 \mathrm{mcg} / \mathrm{mL}$ epinephrine on each side).

- ITM group received a preoperative intrathecal injection of $400 \mu \mathrm{g}$ morphine sulfate .

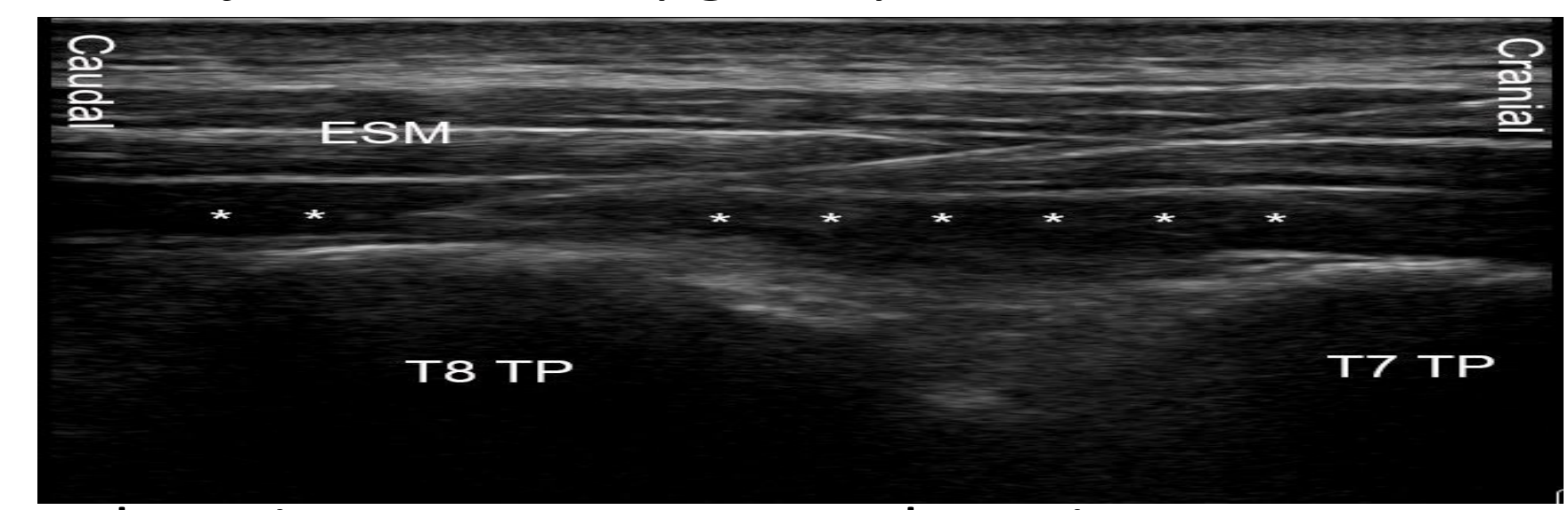

- The primary outcome was the pain score at rest 24 hours after surgery as assessed by an 11-point num eric rating scale.

- The predetermined noninferiority limit was 1.

- Incidences of postoperative nausea/vomiting and pruritus were assessed.

\section{Results}

- The mean resting pain score 24 hours after surgery was $2.5 \pm 1.0$ in the ESP group and $1.3 \pm 1.1$ in the ITM group $(p<0.001)$.

- The mean treatment difference (ESP-ITM) in the 24 -hour pain score was $1.2(95 \%$ confidence interval [Cl]: 0.7 to 1.8$)$, and the upper limit of the $95 \% \mathrm{Cl}(1.8)$ was higher than the prespecified noninferiority margin $(\delta=1)$, indicating that ESP was inferior to ITM.

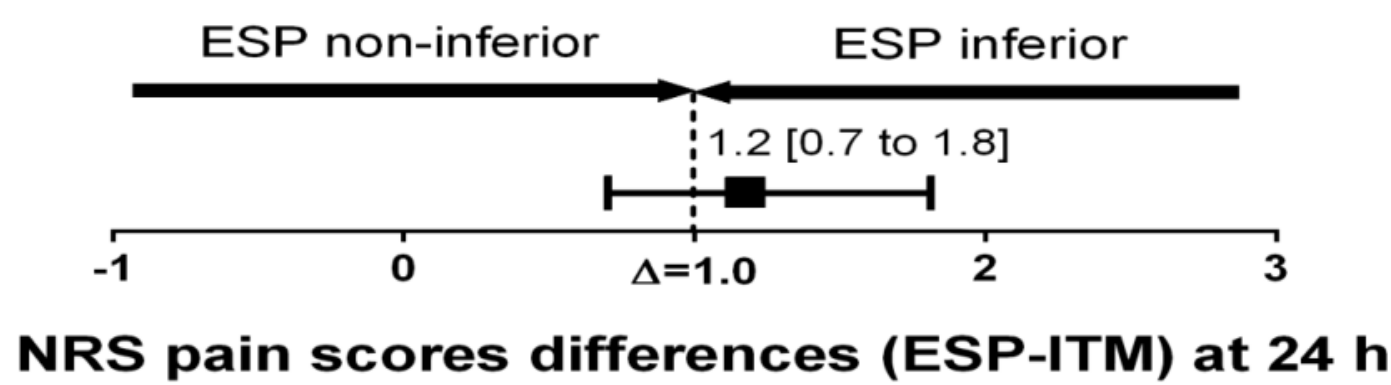

- Pain scores between the groups were similar 48 and 72 hours after the operation.

- There was no significant difference in cumulative opioid consumption at 24,48 , and 72 hours, or patient satisfaction with analgesia.

- The ESP group had a significantly lower incidence of postoperative vomiting [2 (7\%) vs. $13(48 \%)$ patients, $\mathrm{p}=0.002]$ and pruritus [2 (7\%) vs. 25 (93\%) patients, $p<0.001]$.

\section{Conclusions}

- Bilateral single-injection ESP block resulted in higher resting pain scores 24 hours after surgery compared to ITM and thus did not meet the conditions of non-inferiority.

- However, the pain intensity with ESP block was mild (mean $<3 / 10$ ) and associated with reduced incidence of postoperative vomiting and pruritus.

- It may therefore still be considered a viable option for postoperative analgesia after laparoscopic living donor hepatectomy. 